\title{
REALISASI POSITIF STABIL ASIMTOTIK DARI SISTEM LINIER DISKRIT
}

\author{
NOVITA ASWAN \\ Program Studi Magister Matematika, \\ Fakultas Matematika dan Ilmu Pengetahuan Alam, Universitas Andalas, \\ Kampus UNAND Limau Manis Padang, Indonesia, \\ novitaaswan9@gmail.com
}

\begin{abstract}
Abstrak. Diberikan sistem kontrol linier diskrit berikut
$\mathbf{x}(t+1)=A \mathbf{x}(t)+B \mathbf{u}(t)$

$\mathbf{y}(t)=C \mathbf{x}(t)+D \mathbf{u}(t)$

dimana $A \in \mathbb{R}^{n \times n}, B \in \mathbb{R}^{n \times m}, C \in \mathbb{R}^{p \times n}$ dan $D \in \mathbb{R}^{p \times m}$. Dalam sistem diatas, $\mathbf{x}(t) \in \mathbb{R}^{n}$ menyatakan vektor keadaan (state), $\mathbf{u}(t) \in \mathbb{R}^{m}$ menyatakan vektor input (kontrol), $\mathbf{y}(t) \in \mathbb{R}^{p}$ menyatakan vektor output, dan $t \in \mathbb{Z}_{+}$. Dalam tulisan akan dikaji masalah realisasi positif stabil asimtotik dari suatu fungsi transfer dengan pole riil positif untuk sistem SISO. Beberapa contoh disajikan untuk mengilustrasikan hasil utama dalam tulisan ini.
\end{abstract}

Kata Kunci: Sistem Linier Diskrit, Realisasi Positif, Realisasi Positif Stabil Asimtotik, SISO

\section{Pendahuluan}

Jika diberikan suatu sistem kontrol linier, maka dapat ditentukan fungsi transfer yang berkaitan dengan sistem tersebut. Fungsi transfer suatu sistem linier merepresentasikan hubungan antara input dan output sistem tersebut. Akan tetapi, akan menjadi berbeda jika yang terjadi adalah sebaliknya. Jika diberikan suatu fungsi transfer, bagaimanakah bentuk matriks $A, B, C$ dan $D$ yang bersesuaian dengan sistem linier tersebut. Masalah inilah yang disebut realisasi. Menentukan realisasi dari suatu fungsi transfer bukan hal yang mudah, diantaranya pada sistem linier diskrit dengan Single Input dan Single Output (SISO).

\section{Sistem Linier Diskrit}

Sistem linier diskrit ditulis sebagai berikut

$$
\begin{aligned}
\mathbf{x}(t+1) & =A \mathbf{x}(t)+B \mathbf{u}(t), \\
\mathbf{y}(t) & =C \mathbf{x}(t)+D \mathbf{u}(t),
\end{aligned}
$$

dimana $A \in \mathbb{R}^{n \times n}, B \in \mathbb{R}^{n \times m}, C \in \mathbb{R}^{p \times n}$ dan $D \in \mathbb{R}^{p \times m}$. Dalam sistem (2.1), $\mathbf{x}(t) \in \mathbb{R}^{n}$ menyatakan vektor keadaan (state), $\mathbf{u}(t) \in \mathbb{R}^{m}$ menyatakan vektor input (kontrol), $\mathbf{y}(t) \in \mathbb{R}^{p}$ menyatakan vektor output, dan $t \in \mathbb{Z}_{+}$. Fungsi transfer dari sistem (2.1) adalah $T(z)=C\left[I_{n} z-A\right]^{-1} B+D$. 
Definisi 2.1. [2,5] Sistem (2.1) dikatakan positif jika untuk setiap $\mathbf{x}(0) \in \mathbb{R}_{+}^{n}$ dan untuk setiap $\mathbf{u}(t) \in \mathbb{R}_{+}^{m}, t \geq 0$, berlaku $\mathbf{x}(t) \in \mathbb{R}_{+}^{n}$ dan $\mathbf{y}(t) \in \mathbb{R}_{+}^{p}$.

Teorema 2.2. [2,5] Sistem (2.1) adalah positif jika dan hanya jika

$$
A \in \mathbb{R}_{+}^{n \times n}, B \in \mathbb{R}_{+}^{n \times m}, C \in \mathbb{R}_{+}^{p \times n}, D \in \mathbb{R}_{+}^{p \times m} .
$$

Teorema 2.3. [5] Sistem positif (2.1) adalah stabil asimtotik jika dan hanya jika semua nilai eigen $z_{1}, z_{2}, \ldots, z_{n}$ dari matriks $A \in \mathbb{R}_{+}^{n \times n}$ mempunyai modulo yang kurang dari 1, yaitu

$$
\left|z_{i}\right|<1 \text { untuk } i=1,2, \ldots, n \text {. }
$$

Teorema 2.4. [2, 5] Sistem positif (2.1) adalah stabil asimtotik jika dan hanya jika semua koefisien dari polinomial

$$
\begin{aligned}
p_{n}(z) & =\operatorname{det}\left[I_{n}(z+1)-A\right] \\
& =z^{n}+a_{n-1} z^{n-1}+\ldots+a_{1} z+a_{0}
\end{aligned}
$$

adalah positif, yaitu $a_{i}>0 ; i=0,1, \ldots, n-1$.

Untuk sistem dengan single input single output (SISO), fungsi transfer didefinisikan sebagai fungsi $T(z)$ yang memenuhi hubungan

$$
T(z)=\frac{Y(z)}{U(z)}
$$

dimana $Y(z)$ adalah transformasi-z dari sistem output dan $U(z)$ adalah transformasi-z dari sistem input. Selanjutnya, dalam [6] dijelaskan bahwa suatu fungsi transfer $T(z)$ dikatakan proper jika $\lim _{z \rightarrow 0} T(z)=K, K \in \mathbb{R}^{p \times m}$, dan dikatakan strictly proper jika $K=0$.

\section{Realisasi Positif Stabil Asimtotik dari Fungsi Transfer}

Lema 3.1. [6] Matriks-matriks

$$
\begin{aligned}
& \bar{A}_{k}=P A_{k} P^{-1} \in \mathbb{R}_{+}^{n \times n}, \bar{B}_{k}=P B_{k} \in \mathbb{R}_{+}^{n \times m}, \\
& \bar{C}_{k}=C_{k} P^{-1} \in \mathbb{R}_{+}^{p \times n}, \quad \bar{D}_{k}=D_{k} \in \mathbb{R}_{+}^{p \times m}, \quad k=1, \ldots, q .
\end{aligned}
$$

adalah realisasi positif stabil asimtotik dari fungsi transfer proper $T(z) \in \mathbb{R}_{+}^{p \times m}(z)$ untuk sebarang matriks monomial $P \in \mathbb{R}_{+}^{p \times m}$ jika dan hanya jika matriks

$$
A_{k} \in \mathbb{R}_{+}^{n \times n}, B_{k} \in \mathbb{R}_{+}^{n \times m}, C_{k} \in \mathbb{R}_{+}^{p \times n}, D_{k} \in \mathbb{R}_{+}^{p \times m}, \quad k=1, \ldots, q
$$

adalah realisasi positif stabil asimtotik dari $T(z)$.

Diberikan fungsi transfer proper berikut

$$
T(z)=\frac{n(z)}{d(z)}=\frac{b_{n} z^{n}+b_{n-1} z^{n-1}+\ldots+b_{1} z+b_{0}}{z^{n}+a_{n-1}+\ldots+a_{1} z+a_{0}}
$$

yang hanya memiliki pole riil positif dan tidak perlu berbeda, sebutlah $\alpha_{1}, \alpha_{2}, \ldots, \alpha_{n}$. Dari (3.2) diketahui

$$
\begin{aligned}
d_{n}(z) & =\left(z-\alpha_{1}\right)\left(z-\alpha_{2}\right) \ldots\left(z-\alpha_{n}\right) \\
& =z^{n}+a_{n-1} z^{n-1}+\ldots+a_{1} z+a_{0}
\end{aligned}
$$


dimana

$$
\begin{aligned}
a_{n-1} & =-\left(\alpha_{1}+\alpha_{2}+\ldots+\alpha_{n}\right), \\
a_{n-2} & =-\alpha_{1}\left(\alpha_{2}+\alpha_{3}+\ldots+\alpha_{n}\right)-\alpha_{2}\left(\alpha 31+\alpha_{4}+\ldots+\alpha_{n}\right)-\ldots-\alpha_{n-1} \alpha_{n},, \\
\vdots & =\vdots \\
a_{0} & =(-1)^{n} \alpha_{1} \alpha_{2} \ldots \alpha_{n} .
\end{aligned}
$$

Lema 3.2. [6] Jika polinomial

$$
p_{n}(z)=z^{n}+(-1)^{1} \tilde{a}_{n-1} z^{n-1}+(-1)^{2} \tilde{a}_{n-2} z^{n-2}+\ldots+(-1)^{n} \tilde{a}_{0}
$$

hanya memiliki akar riil positif $\alpha_{k}>0, k=1,2, \ldots, n$ maka

$$
\tilde{a}_{n-k}>0 \text { untuk } k=1,2, \ldots, n .
$$

Teorema 3.3. [6] Polinomial

$$
d_{n}(z)=z^{n}+a_{n-1} z^{n-1}+\ldots+a_{1} z+a_{0}
$$

hanya memiliki akar riil positif yang memenuhi

$$
z_{k}<1 \text { untuk } k=1,2, \ldots, n
$$

jika dan hanya jika semua koefisien polinomial

$$
\begin{aligned}
\bar{d}_{n}(z)=d_{n}(z+1) & =(z+1)^{n}+a_{n-1}(z+1)^{n-1}+\ldots+a_{1}(z+1)+a_{0} \\
& =z^{n}+\bar{a}_{n-1} z^{n-1}+\ldots+\bar{a}_{1} z+\bar{a}_{0},
\end{aligned}
$$

dimana

$$
\bar{a}_{n-1}=n+a_{n-1}, \ldots, \bar{a}_{0}=1+a_{0}+a_{1}+\ldots+a_{n-1}
$$

adalah positif, yaitu

$$
\bar{a}_{k}>0 \text { untuk } k=0,1, \ldots, n-1 \text {. }
$$

Teorema 3.4. [6] Terdapat realisasi positif stabil asimtotik

$$
\begin{aligned}
& \bar{A}_{k}=P A_{k} P^{-1} \in \mathbb{R}_{+}^{n \times n}, \bar{B}_{k}=P B_{k} \in \mathbb{R}_{+}^{n \times 1}, \\
& \bar{C}_{k}=C_{k} P^{-1} \in \mathbb{R}_{+}^{1 \times n}, \quad \bar{D}_{k}=D_{k} \in \mathbb{R}_{+}^{1 \times 1}, \quad k=1,2
\end{aligned}
$$

untuk sebarang matriks monomial $P \in \mathbb{R}_{+}^{n \times n}$ dengan $A_{k}, B_{k}, C_{k}$ dan $D_{k}$ berbentuk

$$
\begin{aligned}
A_{1}= & {\left[\begin{array}{ccccc}
\alpha_{1} & 1 & 0 & \ldots & 0 \\
0 & \alpha_{2} & 1 & \ldots & 0 \\
\vdots & \vdots & \vdots & \ldots & \vdots \\
0 & 0 & 0 & \ldots & \alpha_{n}
\end{array}\right], B_{1}=\left[\begin{array}{c}
0 \\
0 \\
\vdots \\
1
\end{array}\right], } \\
C_{1}^{T}= & {\left[\begin{array}{c}
b_{0}-a_{0} b_{n}-\hat{a}_{20} c_{2}-\hat{a}_{30} c_{3}-\ldots-\hat{a}_{n, 0} c_{n} \\
\vdots \\
b_{n-2}-a_{n-2} b_{n}-\hat{a}_{n, n-2} c_{n} \\
b_{n-1}-a_{n-1} b_{n}
\end{array}\right], D_{1}=\left[b_{n}\right] }
\end{aligned}
$$


atau

$$
A_{2}=A_{1}^{T}, \quad B_{2}=C_{1}^{T}, \quad C_{2}=B_{1}^{T}, \quad D_{2}=D_{1},
$$

dari fungsi transfer (3.2) dengan pole riil positif $\alpha_{1}, \alpha_{2}, \ldots, \alpha_{n}$ jika dan hanya jika $C_{1}^{T} \succeq 0$, dimana

$$
\begin{aligned}
\hat{a}_{20} & =-\alpha_{1}, \\
\hat{a}_{30} & =\alpha_{1} \alpha_{2} \\
\hat{a}_{n, 0} & =(-1)^{n-1} \alpha_{1} \alpha_{2} \ldots \alpha_{n-1}, \ldots, \\
\hat{a}_{31} & =-\left(\alpha_{1} \alpha_{2}\right), \ldots, \\
\hat{a}_{n, n-2} & =-\left(\alpha_{1}+\alpha_{2}+\alpha_{3}+\ldots+\alpha_{n-1}\right) .
\end{aligned}
$$

Bukti. Matriks $A_{1} \in \mathbb{R}_{+}^{n \times n}$ adalah stabil asimtotik jika dan hanya jika nilai-nilai eigennya, yaitu $z_{k}=\alpha_{k}, k=1,2, \ldots, n$ hanya riil positif dan memenuhi syarat (3.4). Matriks

$$
D_{1}=\lim _{z \rightarrow 0} T(z)=\left[b_{n}\right] \in \mathbb{R}_{+}^{1 \times 1}
$$

jika dan hanya jika $b_{n} \geq 0$. Fungsi transfer stricly proper berbentuk

$$
\begin{aligned}
T_{s p}(z) & =T(z)-D_{1} \\
& =\frac{\bar{b}_{n-1} z^{n-1}+\ldots+\bar{b}_{1} z+\bar{b}_{0}}{z^{n}+a_{n-1} z^{n-1}+\ldots+a_{1} z+a_{0}},
\end{aligned}
$$

dimana $\bar{b}_{k}=b_{k}-a_{k} b_{n}$ untuk $k=0,1, \ldots, n-1$.

$$
\begin{aligned}
& \text { Dengan mengasumsikan } B_{1}=\left[\begin{array}{c}
0 \\
0 \\
\vdots \\
1
\end{array}\right] \in \mathbb{R}_{+}^{n \times 1} \text {, diperoleh } \\
& T_{s p}(z)=C_{1}\left[I_{n} z-A_{1}\right]^{-1} B_{1} \\
& =\left[c_{1} \ldots c_{n}\right]\left[\begin{array}{ccccc}
z-\alpha_{1} & -1 & 0 & \ldots & 0 \\
0 & z-\alpha_{2} & -1 & \ldots & 0 \\
\vdots & \vdots & \vdots & \ldots & \vdots \\
0 & 0 & 0 & \ldots & z-\alpha_{n}
\end{array}\right]^{-1}\left[\begin{array}{c}
0 \\
0 \\
\vdots \\
1
\end{array}\right] \\
& =\frac{1}{d_{n}(z)}\left[c_{1} \ldots c_{n}\right]\left[\begin{array}{c}
p_{1}(z) \\
p_{2}(z) \\
\vdots \\
p_{n}(z)
\end{array}\right] \\
& =\frac{c_{1} p_{1}(z)+c_{2} p_{2}(z)+\ldots+c_{n} p_{n}(z)}{d_{n}(z)} \text {, }
\end{aligned}
$$


dimana

$$
\begin{aligned}
d_{n}(z) & =\left(z-\alpha_{1}\right)\left(z-\alpha_{2}\right) \ldots\left(z-\alpha_{k}\right) \\
& =z^{n}+(-1)^{1} \tilde{a}_{n-1} z^{n-1}+(-1)^{2} \tilde{a}_{n-2} z^{n-2}+\ldots+(-1)^{n} \tilde{a}_{0} . \\
p_{1}(z) & =1 \\
p_{2}(z) & =z-\alpha_{1}=z+\tilde{a}_{20}, \quad \text { dimana } \tilde{a}_{20}=-\alpha_{1} \\
p_{3}(z) & =\left(z-\alpha_{1}\right)\left(z-\alpha_{2}\right) \\
& =z^{2}+\tilde{a}_{31} z+\tilde{a}_{30}, \quad \text { dimana } \tilde{a}_{31}=-\left(\alpha_{1}+\alpha_{2}\right), \tilde{a}_{30}=\alpha_{1} \alpha_{2}, \\
\vdots & \vdots \\
p_{n}(z) & =\left(z-\alpha_{1}\right)\left(z-\alpha_{2}\right) \ldots\left(z-\alpha_{n-1}\right) \\
& =z^{n-1}+\tilde{a}_{n, n-2} z^{n-2}+\ldots+\tilde{a}_{n, 1} z+\tilde{a}_{n, 0},
\end{aligned}
$$

dimana

$$
\begin{aligned}
\tilde{a}_{n, n-2} & =-\left(\alpha_{1}+\alpha_{2}+\ldots+\alpha_{n-1}\right), \ldots, \\
\tilde{a}_{n, 0} & =(-1)^{n-1} \alpha_{1} \alpha_{2} \ldots \alpha_{n-1} .
\end{aligned}
$$

Dengan membandingkan persamaan (3.10) dan (3.11) diperoleh

$$
\begin{aligned}
& c_{n}=\bar{b}_{n-1}=b_{n-1}-a_{n-1} b_{n}, \\
& c_{n-1}=\bar{b}_{n-2}-\tilde{a}_{n, n-2} c_{n}=b_{n-2}-a_{n-2} b_{n}-\tilde{a}_{n, n-2} c_{n}, \\
& \vdots \\
& c_{1}=\bar{b}_{0}-\tilde{a}_{20} c_{2}-\tilde{a}_{30} c_{3}-\ldots-\tilde{a}_{n, 0} c_{n} \\
& \quad=b_{0}-a_{0} b_{n}-\tilde{a}_{20} c_{2}-\tilde{a}_{30} c_{3}-\ldots-\tilde{a}_{n, 0} c_{n} .
\end{aligned}
$$

Dari persamaan (3.12) diperoleh bahwa $C_{1} \in \mathbb{R}_{+}^{1 \times n}$ jika dan hanya jika $C_{1} \succeq 0$ dimana (3.9) terpenuhi. Dengan menggunakan kesamaan

$$
\begin{aligned}
T(z) & =[T(z)]^{T} \\
& =\left[C_{1}\left[I_{n} z-A_{1}\right]^{-1} B_{1}+D_{1}\right]^{T} \\
& =C_{1}^{T}\left[I_{n} z-A_{1}^{T}\right]^{-1} B_{1}^{T}+D_{1} \\
& =B_{1}^{T}\left[I_{n} z-A_{1}^{T}\right]^{-1} C_{1}^{T}+D_{1} \\
& =C_{2}\left[I_{n} z-A_{2}\right]^{-1} B_{2}+D_{2} .
\end{aligned}
$$

diperoleh matriks-matriks (3.8). Berdasarkan Lema 3.1, matriks-matriks (3.6) adalah suatu realisasi positif stabil asimtotik untuk sebarang matriks monomial $P \in \mathbb{R}_{+}^{n \times n}$ jika dan hanya jika (3.7) atau (3.8) adalah realisasi positif stabil asimtotik dari $T(z)$.

\section{Contoh Menentukan Realisasi Positif Stabil Asimtotik dari Sistem Linier Diskrit}

Tentukan realisasi dari fungsi transfer berikut.

$$
T(z)=\frac{0.1 z^{3}+z^{2}+2 z+3}{z^{3}-1.1 z^{2}+0.35 z-0.025} .
$$


40 Novita Aswan

Dari fungsi transfer $T(z)$ di atas diketahui bahwa

$$
\begin{aligned}
d_{3}(z) & =z^{3}-1.1 z^{2}+0.35 z-0.025, \\
& =(z-0.1)\left(z^{2}-z+0.25\right), \\
& =(z-0.1)(z-0.5)(z-0.5),
\end{aligned}
$$

dengan $\tilde{a}_{2}=1.1>0, \tilde{a}_{1}=0.35>0$ dan $\tilde{a}_{0}=0.025>0$. Selanjutnya,

$$
\begin{aligned}
\bar{d}_{3}(z) & =d_{3}(z+1) \\
& =(z+1)^{3}-1.1(z+1)^{2}+0.35(z+1)-0.025 \\
& =(z+1)\left(z^{2}+2 z+1\right)-1.1 z^{2}-2.2 z-1.1+0.35 z+0.35-0.025 \\
& =z^{3}+3 z^{2}+3 z+1-1.1 z^{2}-1.85 z-0.775 \\
& =z^{3}+1.9 z^{2}+1.15 z+0.225
\end{aligned}
$$

sehingga semua koefisien dari $\bar{d}_{3}(z)$ adalah positif, yaitu $\bar{a}_{0}=0.225>0, \bar{a}_{1}=$ $1.15>0, \bar{a}_{2}=1.9>0$ dan $\bar{a}_{3}=1>0$. Berikutnya, diperoleh

$$
\begin{aligned}
& c_{3}=b_{2}-a_{2} b_{3}=1+0.11=1.11>0, \\
& c_{2}=b_{1}-a_{1} b_{3}-\hat{a}_{31} c_{3}=2-0.035+1.11=3.075>0,
\end{aligned}
$$

dimana $\hat{a}_{31}=-\left(\alpha_{1}+\alpha_{2}\right)=-(0.5+0.5)=-1$ dan

$$
\begin{aligned}
c_{3} & =b_{0}-a_{0} b_{3}-\hat{a}_{20} c_{2}-\hat{a}_{30} c_{3} \\
& =3+0.0025+1.5375-0.2775=4.2625>0
\end{aligned}
$$

dimana $\hat{a}_{2}=-\alpha_{1}=-0.5$ dan $\hat{a}_{30}=\alpha_{1} \alpha_{2}=0.25$. Dengan demikian,

$$
A_{1}=\left[\begin{array}{ccc}
0.5 & 1 & 0 \\
0 & 0.5 & 1 \\
0 & 0 & 0.1
\end{array}\right], \quad B_{1}=\left[\begin{array}{l}
0 \\
0 \\
1
\end{array}\right], \quad C_{1}=\left[\begin{array}{ll}
4.26253 .075 & 1.11
\end{array}\right], \quad D_{1}=[0.1]
$$

Selanjutnya, matriks realisasi positif stabil asimtotik dari $T(z)$ adalah

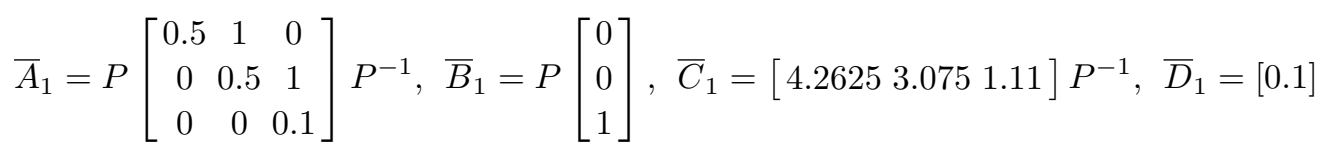




$$
\begin{aligned}
& \text { Misalkan terdapat } P=\left[\begin{array}{lll}
2 & 0 & 0 \\
0 & 1 & 0 \\
0 & 0 & 1
\end{array}\right] \text { dengan } P^{-1}=\left[\begin{array}{ccc}
0.5 & 0 & 0 \\
0 & 1 & 0 \\
0 & 0 & 1
\end{array}\right] \text {. Maka, } \\
& \bar{A}_{1}=\left[\begin{array}{lll}
2 & 0 & 0 \\
0 & 1 & 0 \\
0 & 0 & 1
\end{array}\right]\left[\begin{array}{ccc}
0.5 & 1 & 0 \\
0 & 0.5 & 1 \\
0 & 0 & 0.1
\end{array}\right]\left[\begin{array}{ccc}
0.5 & 0 & 0 \\
0 & 1 & 0 \\
0 & 0 & 1
\end{array}\right] \\
& =\left[\begin{array}{lll}
2 & 0 & 0 \\
0 & 1 & 0 \\
0 & 0 & 1
\end{array}\right]\left[\begin{array}{ccc}
0.25 & 1 & 0 \\
0 & 0.5 & 0 \\
0 & 0 & 0.1
\end{array}\right] \\
& =\left[\begin{array}{ccc}
0.5 & 2 & 0 \\
0 & 0.5 & -1 \\
0 & 0 & 0.1
\end{array}\right] \\
& \bar{B}_{1}=\left[\begin{array}{lll}
2 & 0 & 0 \\
0 & 1 & 0 \\
0 & 0 & 1
\end{array}\right]\left[\begin{array}{l}
0 \\
0 \\
1
\end{array}\right]=\left[\begin{array}{l}
0 \\
0 \\
1
\end{array}\right] \text {, } \\
& \bar{C}_{1}=\left[\begin{array}{lll}
4.2625 & 3.075 & 1.11
\end{array}\right]\left[\begin{array}{rrr}
0.5 & 0 & 0 \\
0 & 1 & 0 \\
0 & 0 & 1
\end{array}\right]=\left[\begin{array}{lll}
2.13125 & 3.075 & 1.11
\end{array}\right] \text {, } \\
& \bar{D}_{1}=[0.1] .
\end{aligned}
$$

Perhatikan bahwa

$$
\begin{aligned}
& T(z)=\bar{C}_{1}\left[I_{3} z-\bar{A}_{1}\right]^{-1} \bar{B}_{1}+\bar{D}_{1} \\
& =\left[\begin{array}{lll}
2.13125 & 3.075 & 1.11
\end{array}\right]\left[\begin{array}{ccc}
z-0.5 & -2 & 0 \\
0 & z-0.5 & -1 \\
0 & 0 & z-0.1
\end{array}\right]^{-1}\left[\begin{array}{l}
0 \\
0 \\
1
\end{array}\right]+[0.1] \\
& =\frac{1}{z^{3}-1.1 z^{2}+0.35 z-0.025}[2.131253 .0751 .11] \times \\
& {\left[\begin{array}{c}
2 \\
z-0.5 \\
z^{2}-z+0.25
\end{array}\right]+[0.1]} \\
& =\frac{1.11 z^{2}+1.965 z+3.0025+0.1 z^{3}-0.11 z^{2}+0.035 z-0.0025}{z^{3}-1.1 z^{2}+0.35 z-0.025} \\
& =\frac{0.1 z^{3}+z^{2}+2 z+3}{z^{3}-1.1 z^{2}+0.35 z-0.025} \text {. }
\end{aligned}
$$

\section{Ucapan Terima Kasih}

Penulis mengucapkan terima kasih kepada Bapak Muhafzan, Bapak Mahdhivan Syafwan, Bapak Syafrizal Sy, Bapak Admi Nazra, dan Ibu Yanita yang telah memberikan masukan dan saran, sehingga tulisan ini dapat diselesaikan dengan baik. 
42 Novita Aswan

\section{Daftar Pustaka}

[1] Anton, H. 1991. Aljabar Linier Elementer Edisi Kedelapan-Jilid 1. Erlangga, Jakarta

[2] Farina, L and Rinaldi, S. 2000. Positive Linear Systems. John Wiley and Sons, New York

[3] Kaczorek, T. 1991. Linear Control System. Vol.1. Research Studies Press, England

[4] Kaczorek, T. 2002. Positive $1 D$ and 2D Systems. Springer-Verlag, London

[5] Kaczorek, T. 2012. Positive Stable Realizations of Discrete-time Linear System. Buletin of the Polish Academy of Sciences, Vol. 60, No. 3

[6] Ogata, K. 1995. Discrete-Time Control Systems. Prentice Hall, New Jersey 